\title{
Comportement du limon de Jossigny remanié soumis à une pression interstitielle négative
}

\section{Behavior of remolded Jossigny loam under negative pore pressure}

\author{
J.-M. FLEUREAU \\ Chef de Travaux, Ecole centrale de Paris* \\ INDARTO \\ Chercheur, Ecole centrale de Paris*
}

Rev. Franç. Géotech., n62, pp. 59.66 (janvier 1993)

\section{Résumé}

L'étude expérimentale du comportement du limon de Jossigny $\left(w_{L}=37 ; I_{p}\right.$ $=19$ ) sur différents chemins à pression interstitielle imposée (drainage-humidification, chemins triaxiaux normalement consolidés ou surconsolidés) montre que la logique des sols saturés et la notion classique de contrainte effective de Terzaghi restent valables tant que l'eau est la phase continue, c'està-dire, en première approximation, pour des teneurs en eau supérieures à la limite de retrait.

\begin{abstract}
The experimental study of the behavior of Jossigny loam $\left(w_{L}=37 ; I_{p}=19\right)$ on several pressure-controlled paths (drying-wetting, normally consolidated and overconsolidated triaxial paths) shows that the logic of saturated soils and the usual concept of Terzaghi's effective stress remain valid as long as water is the continuous phase, i.e. approximately for water contents larger than the shrinkage limit.
\end{abstract}

\footnotetext{
- Laboratoire de Mécanique des sols, structures et matériaux, CNRS URA 850 et GRECO 90 « Géomatériaux *, Grande Voie des vignes, 92295 Chatenay-Malabry.
} 


\section{INTRODUCTION}

Le limon de Jossigny a été choisi, dans le cadre du groupe "sols non saturés " du GRECO Géomatériaux, comme matériau de référence pour comprendre et modéliser le comportement des sols à pression négative, compte tenu de l'important travail de caractérisation physique et mécanique déjà réalisé par les laboratoires des Ponts et Chaussées sur le site. En outre, un grand nombre d'essais ont été effectués par le CERMES de I'ENPC sur le sol compacté à l'Optimum Proctor Normal (DELAGE et al. 1987). Il nous a paru intéressant de compléter ces travaux en étudiant le comportement du sol, initialement saturé, sur des chemins de drainage et d'humidification.

Dans le domaine étudié (jusqu'à $800 \mathrm{kPa}$ de pression négative), le sol ne se désature que relativement peu. On retrouve assez fréquemment ce type de comportement dans la pratique, dans les régions tempérées et notamment en France, où les conditions climatiques entraînent rarement une désaturation du sol au-delà des premiers centimètres. Dans ce cadre, l'étude entreprise avait pour but de comparer les comportements du sol à pression positive et négative, tant du point de vue des variations de volume que de la résistance, et d'examiner si la notion de contrainte effective de Terzaghi restait toujours valable dans ce domaine.

\section{MATÉRIAU ET MÉTHODES DE MESURE}

Le matériau étudié est le limon prélevé sur le site de Jossigny, à l'est de Paris. Il s'agit en fait d'une argile peu plastique (classée Ap). Ses principales caractéristiques sont indiquées dans le tableau 1.

La courbe de drainage-humidification a été déterminée en utilisant les techniques classiques d'imposition de la pression interstitielle négative et en mesurant les caractéristiques - volume, teneur en eau - des échantillons à l'équilibre (BIAREZ et al. 1988). Quelle que soit la méthode employée (plaques tensiométriques, surpres. sion d'air, osmose, solutions salines, etc.), les résultats peuvent toujours être exprimés en fonction d'une pression négative d'eau équivalente $-\mathrm{u}_{w}$, ou de son lo. garithme, le $\mathrm{pF}\left(\mathrm{pF}=\log \left(-\mathrm{u}_{\omega}\right)\right.$, $\mathrm{u}_{\omega}$ en $\mathrm{cm}$ de colonne d'eau).

Les essais triaxiaux à pression négative imposée ont été faits dans une cellule triaxiale modifiée (INDARTO, 1991), dans laquelle une membrane cellulosique semiperméable est placée à la base de l'échantillon. On impose à celui-ci une pression interstitielle d'eau nulle.
L'échantillon ( $\mathrm{H}=35 \mathrm{~mm}, \mathrm{D}=35 \mathrm{~mm}$ ), sans membrane imperméable, est soumis par ailleurs sur ses faces latérales à une pression d'air $\mathrm{u}_{o}$, qui joue le rôle d'une pression de confinement et permet en même temps d'imposer à l'intérieur de l'échantillon, la différence de pression $u_{a}-u_{w}$ qui conditionne l'équilibre hydrique du matériau. Les conditions de ces essais drainés peuvent se résumer à :

$$
\sigma_{3}=\mathrm{u}_{\mathrm{o}} ; \mathrm{u}_{\mathrm{w}}=0 ; \mathrm{u}_{a}-\mathrm{u}_{\mathrm{w}}=\mathrm{u}_{a} \text {. }
$$

Pendant l'écrasement, effectué à une vitesse de $2,5 \mu \mathrm{m} /$ $\min (0,008 \% / \mathrm{min})$, on mesure la force d'écrasement, le déplacement vertical et les variations de périmètre de l'échantillon.

L'essai de drainage a été effectué sur des échantillons préparés sous forme de pâte à une teneur en eau égale à une fois et demi la limite de liquidité du matériau. La pâte, sêchée à l'air, a été progressivement réhumidifiée sur le chemin d'humidification. Pour les essais triaxiaux, la pâte a été initialement consolidée dans les conditions cedométriques sous une contrainte d'environ $100 \mathrm{kPa}$, toujours à l'état saturé. Dans le cas des essais consolidés non drainés saturés, les échantillons ont été soumis ensuite à une consolidation isotrope. Pour les essais à pression négative imposée, deux séries d'échantillons ont été préparées après consolidation:

- les premiers ( « normalement consolidés ») ont été drainés par application de la pression d'air jusqu'à la valeur de $\mathrm{u}_{0}-\mathrm{u}_{\omega}$ choisie, puis écrasés dans ces conditions ;

- les seconds ("surconsolidés ») ont d'abord été drainés jusqu'à une pression d'air de $800 \mathrm{kPa}$, puis réhumidifiés par abaissement de la pression $\mathrm{u}_{a}-\mathrm{u}_{u}$ jusqu'à la valeur choisie pour l'essai. Le rapport de surconsolidation hydrique (OCR) est le rapport de la pression négative maximale à la pression négative actuelle.

\section{RÉSULTATS ET DISCUSSION}

\subsection{Comportement du sol sur chemin de drainage-humidification à contrainte nulle}

Les résultats de l'essai de drainage-humidification sont représentés sur la figure 1, où l'indice des vides, le degré de saturation et la teneur en eau sont tracés en fonction de la pression négative d'eau $-\mathrm{u}_{\omega}$ ou du $\mathrm{pF}$ dans la partie droite de la figure, et en fonction de la teneur en eau dans la partie gauche. Il s'agit là d'une représentation globale de l'état du sol permettant de bien

Tableau 1. - Caractéristiques du limon de Jossigny

\begin{tabular}{|c|c|c|c|c|c|c|c|c|}
\hline \multicolumn{3}{|c|}{ Granulométrie } & \multicolumn{3}{c|}{ Plasticité } & \multicolumn{2}{c|}{ Compactage } & Densité grains \\
\hline$\%<80 \mu \mathrm{m}$ & $\%<2 \mu \mathrm{m}$ & $\mathrm{D}_{50}$ & $\mathrm{~W}_{\mathrm{L}}$ & $\mathrm{W}_{\mathrm{p}}$ & $\mathrm{I}_{\mathrm{p}}$ & $\mathrm{W}_{\mathrm{OPN}}$ & $\gamma_{\text {dopN }} / \gamma_{\mathrm{w}}$ & $\gamma_{\mathrm{s}} / \gamma_{\mathrm{w}}$ \\
\hline 80 & 28 & $20 \mu \mathrm{m}$ & 37 & $16-19$ & $18-21$ & 15,5 & 1,75 & 2,74 \\
\hline
\end{tabular}



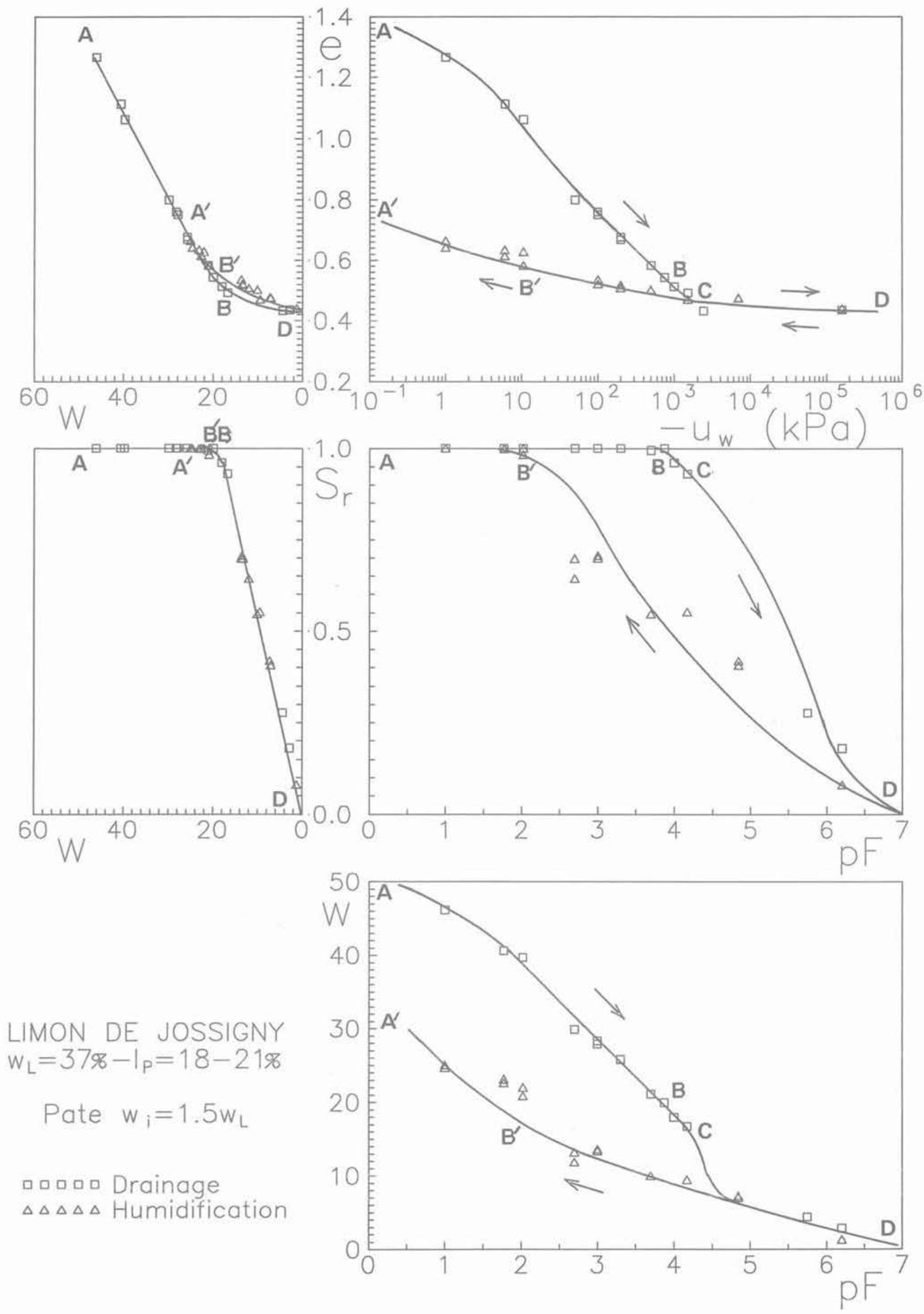

Fig. 1 - Essai de drainage-humidification sur le limon de Jossigny initialement saturé $\left(w_{1}=1,5 w_{\mathrm{L}}\right)$. Fig. 1 - Drying-wetting test on Jossigny loam, initially saturated $(w=1.5 \mathrm{w})$. 
suivre l'évolution simultanée des différents paramètres. On retrouve les différentes phases de comportement déjà observées sur de nombreux matériaux argileux (BIAREZ et al. 1988, 1989; FLEUREAU et al. 1990): - du point initial (A) au point d'entrée d'air (B), le matériau reste saturé, son comportement est identique à celui d'un sol saturé normalement consolidé soumis à une contrainte isotrope (fig. 2). Dans ce domaine, le concept de contrainte effective de Terzaghi reste applicable. Il faut noter la valeur élevée de la pression de désaturation $(-800 \mathrm{kPa})$ pour ce "limon *;

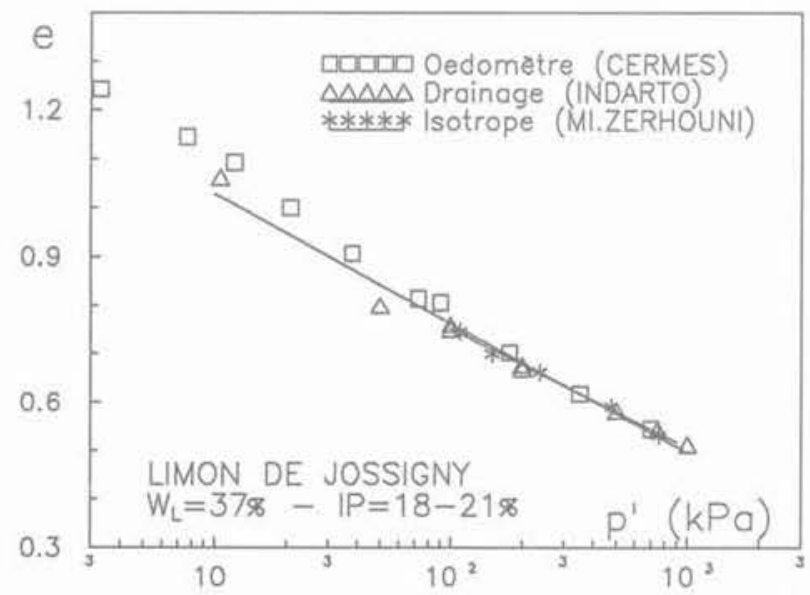

Fig. 2. - Comparaison du chemin de drainage et des chemins de compression isotrope et cedométrique (normalement consolidés) dans le domaine saturé.

Fig. 2 - Comparison between drying and (normally consolidated) isotropic or cedometric compression paths, in the saturated range.

- à partir du point $\mathrm{B}$, le sol se désature rapidement jusqu'à ce que la phase " eau * devienne discontinue (point C). L'eau forme alors des ponts capillaires aux points de contact entre particules, avec de très fortes pressions négatives à l'intérieur des ménisques. Dans ces conditions, les contraintes exercées par l'eau sur les particules sont normales aux plans de contact et ne peuvent entraîner de réorganisation du matériau. On constate, en effet, qu'à partir du point $\mathrm{C}$, l'indice des vides reste constant. La teneur en eau correspondante a le sens d'une limite de retrait;

- entre les points $C$ et $D$, le drainage se poursuit dans un sol pratiquement rigide. Dans ce domaine, la perméabilité au sens de Darcy est globalement nulle, les échanges s'effectuant essentiellement en phase vapeur par diffusion. Il faut noter que, bien que l'augmentation de pression négative n'ait pas d'influence sur les variations de volume dans ce domaine, elle n'en continue pas moins à accroître les forces de contact entre grains et à rigidifier le sol.

On retrouve essentiellement les mêmes phases lors de l'humidification :

- de D à C', un remplissage des pores du milieu rigide. L'hystérésis des degrés de saturation entre drainage et humidification est due essentiellement à la topologie du milieu poreux (effet ink bottle) et, accessoirement, à l'hystérésis des angles de contact
- entre C' et B', le sol se resature avec de faibles variations de volume;

- enfin, entre B' (point de resaturation) et A' (point final), on retrouve le comportement classique d'un sol saturé surconsolidé. Comme au drainage, on peut montrer que, lorsque le degré de saturation est supérieur à 0,9 , la pression négative est équivalente à une contrainte isotrope (fig. 3 ).

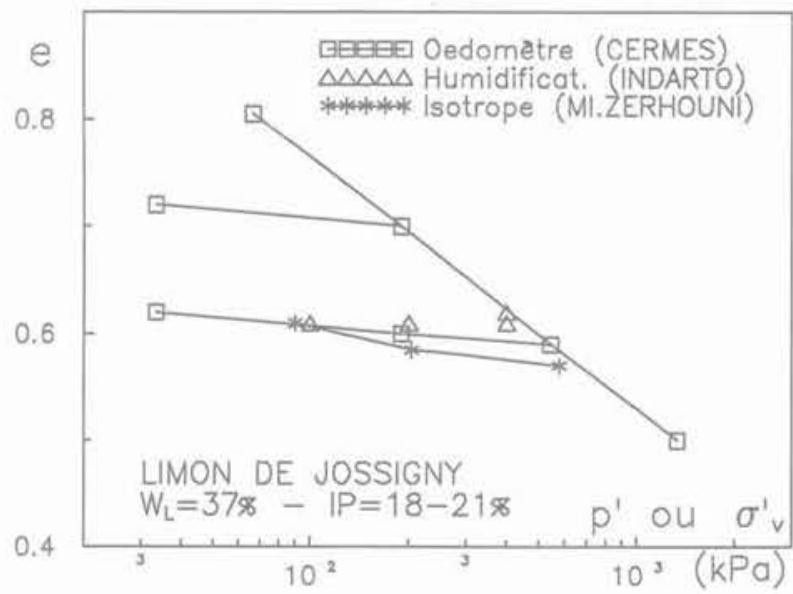

Fig. 3 - Comparaison du chemin d'humidification et du chemin de décompression isotrope (surconsolidé) dans le domaine saturé.

Fig. 3 - Comparison between wetting and (overconsolidated) isotropic or oedometric decompression paths, in the saturated range.

\subsection{Comportement du sol saturé sur chemin triaxial NCU}

Trois essais triaxiaux ont été effectués sur des échantillons saturés normalement consolidés isotropiquement à des contraintes effectives de 100,200 et $400 \mathrm{kPa}$ (avec contrepression de $200 \mathrm{kPa}$ ), puis écrasés en conditions non drainées avec mesure de $u_{\text {. }}$. Les courbes tracées en contraintes effectives (fig. 4) présentent l'allure habituelle de ce type d'essais et l'on peut définir le critère de rupture dans le plan [p'; q'], avec une cohésion nulle et une pente $M=1,3$. Cette valeur est en accord avec l'angle de frottement interne de $31^{\circ}$ mesuré par MESTAT en 1990 au LCPC lors d'une campagne d'essais effectuée sur ce matériau.

Dans le plan [p'; e], les points initiaux des essais apparaissent légèrement décalés par rapport à la courbe de consolidation isotrope, surtout en ce qui concerne l'essai à $100 \mathrm{kPa}$. Pour cet essai, on peut supposer que la contrainte isotrope est trop peu différente de la contrainte odométrique initiale de consolidation et que l'échantillon n'est pas vraiment dans un état normalement consolidé. Néanmoins, les points finaux des trois essais permettent de définir la ligne de plasticité parfaite, parallèle à la droite de consolidation isotrope dans le plan $\left[\log \left(\mathrm{p}^{\prime}\right) ; \mathrm{e}\right]$.

\subsection{Comportement du sol sur chemin triaxial à pression négative}

Les conditions initiale et finale de ces deux séries d'essais sont indiquées dans le tableau 2. 

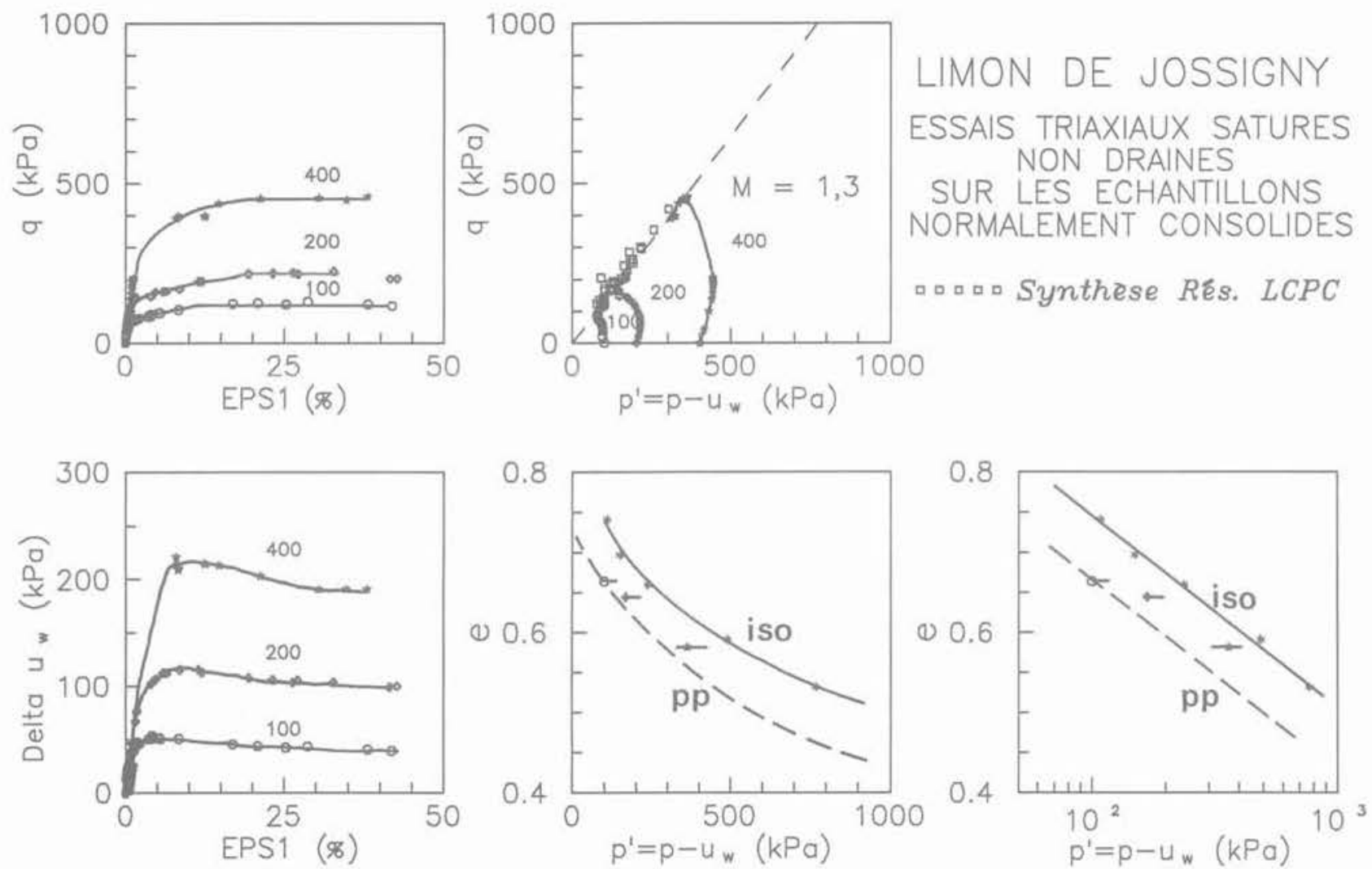

Fig. 4 - Chemin triaxial consolidé non drainé sur le sol saturé à différentes contraintes isotropes initiales.

Fig. 4 - Consolidated undrained triaxial path on saturated soil at several initial isotropic stresses.

Tableau 2. - Conditions initiale et finale des essais triaxiaux à pression négative imposée.

\begin{tabular}{|r|c|c|c|c|c|c|}
\hline Essai & $\mathrm{W}_{i}$ & $\mathrm{~S}_{r i}$ & $\mathrm{~W}_{t}$ & $\mathrm{~S}_{\mathrm{r}}$ & $\mathrm{P}_{\circ}^{\prime}$ & OCR \\
\hline NC 100 & 25,1 & 1 & 18,1 & 1 & 100 & 1 \\
NC 200 & 25,0 & 1 & 19,8 & 1 & 200 & 1 \\
NC 400 & 27,0 & 1 & 22,0 & 1 & 400 & 1 \\
NC 800 & 19,9 & 0,97 & 14,5 & 0,77 & 800 & 1 \\
\hline SC 100 & 19,4 & 0,98 & 18,4 & 0,97 & 100 & 8 \\
SC 200 & 19,0 & 0,96 & 19,1 & 0,97 & 200 & 4 \\
SC 400 & 20,0 & 1 & 20,9 & 0,97 & 400 & 2 \\
\hline
\end{tabular}

Dans le cas des essais normalement consolidés, les échantillons drainés à 100,200 et $400 \mathrm{kPa}$ restent sa. turés, tandis que l'échantillon drainé à $800 \mathrm{kPa}$ se désature légèrement au drainage, puis nettement au cours de l'écrasement. C'est en effet autour de cette valeur de $800 \mathrm{kPa}$ que se situe la pression de désaturation du limon (cf. 3.1). Les résultats des essais NC sont représentés sur la figure 5. Les essais sont drainés (pression d'eau imposée), et on retrouve l'allure des courbes contraintes-déformations ainsi que le comportement contractant d'un sol normalement consolidé. Dans le plan [p'; q], où p' représente la pression moyenne effective, définie par:

$$
\mathrm{p}^{\prime}=\left(\sigma_{1}+2 \sigma_{3}\right) / 3-\mathrm{u}_{w}=\left(\sigma_{1}+2 \mathrm{u}_{\mathrm{n}}\right) / 3
$$

les chemins se terminent sur le même critère de plasticité parfaite que les essais saturés, à l'exception de l'essai NC 800 qui casse rapidement. Le comportement de ce dernier échantillon est typique des matériaux non saturés dans lesquels une rupture prématurée empêche le plus souvent d'atteindre la plasticité parfaite. La non. saturation du sol se traduit en général par un comportement fragile dont on peut essayer de rendre compte par une approche semi-empirique telle que celles proposées par ESCARIO et SAEZ (1986) ou FREDLUND et al. (1987). En fait, on détermine alors une ligne de rupture et non pas un critère de plasticité parfaite.

Dans le plan [p'; e], les points initiaux des essais se situent assez bien sur la courbe de drainage, à l'exception de l'essai NC 400 qui apparait nettement moins dense. Les points finals des trois premiers essais se placent cependant correctement sur la courbe de plasticité parfaite définie par les essais saturés. Une certaine dispersion des résultats apparaît inévitable, quant aux mesures de variations de volume qui sont très délicates à effectuer. Notons que les mesures faites sous contrainte à l'intérieur de la cellule ont été contrôlées par des mesures très précises faites à lextérieur avant la mise en place des échantillons et après la fin des essais.

Les résultats relatifs aux échantillons surconsolidés sont représentés sur la figure 6. Les courbes contraintes-déformations présentent un pic qui correspond, dans le plan $\left[\varepsilon_{1} ; e\right]$, au comportement habituel, d'abord contractant puis dilatant, des sols surconsolidés. Dans le plan $\left[\mathrm{p}^{2} ; \mathrm{q}\right]$, on constate que, pour l'échantillon fortement surconsolidé (SC 100: OCR $=8$ ), le chemin passe par un maximum situé au-dessus de la droite de 

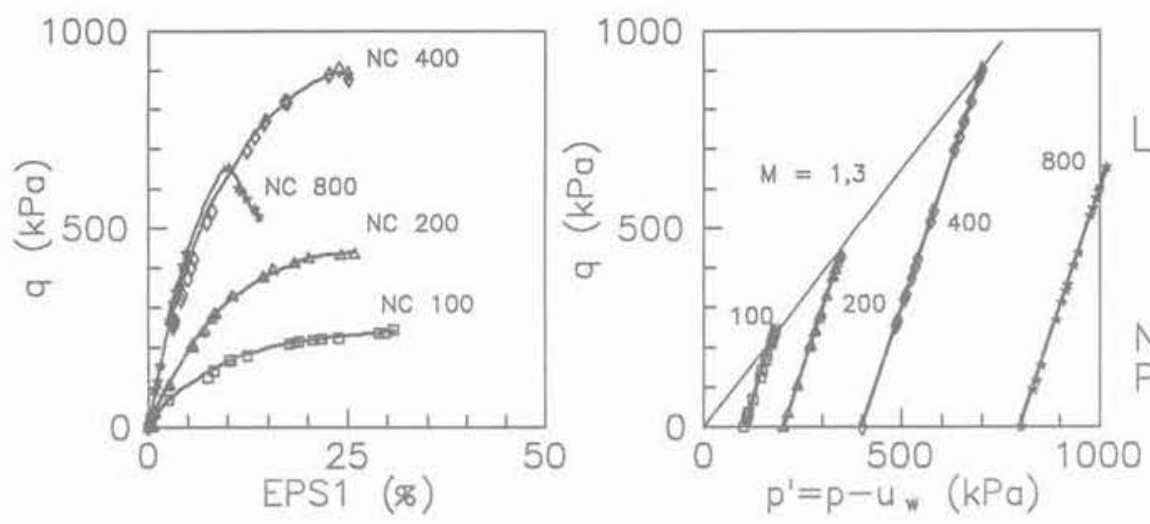

\section{LIMON DE JOSSIGNY}

ESSAIS TRIAXIAUX

A SURPRESSION D'AIR

SUR LES ECHANTILLONS

NORMALEMENT CONSOLIDES

PREPARES PAR DRAINAGE
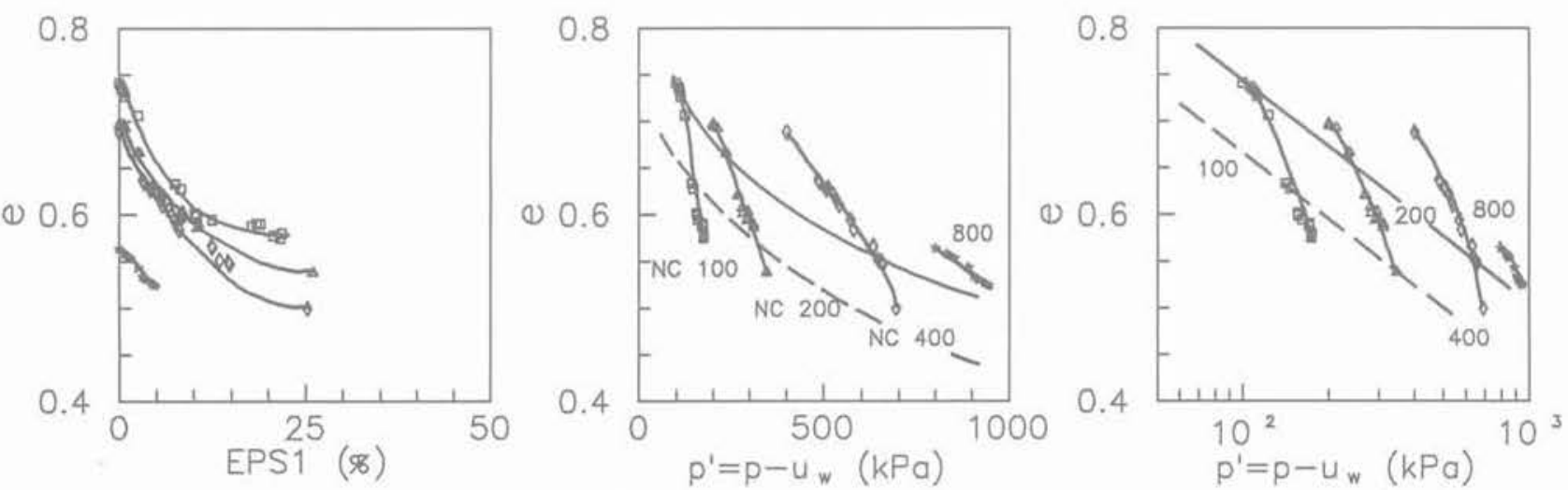

Fig. 5 - Chemin triaxial à pression négative imposée sur le limon de Jossigny normalement consolidé par drainage.

Fig. 5 - Triaxial path on Jossigny loam. normally consolidated by drying. for several controlled negative pressures.
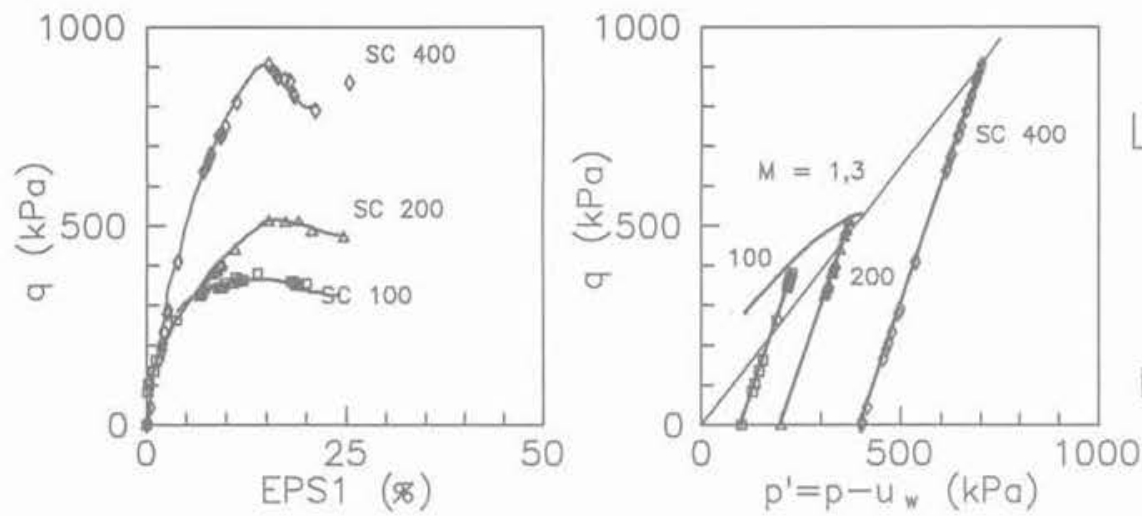

\section{LIMON DE JOSSIGNY} ESSAIS TRIAXIAUX

A SURPRESSION D'AIR

SUR LES ECHANTILLONS SURCONSOLIDES PREPARES PAR DRAINAGE-HUMIDIFICATION
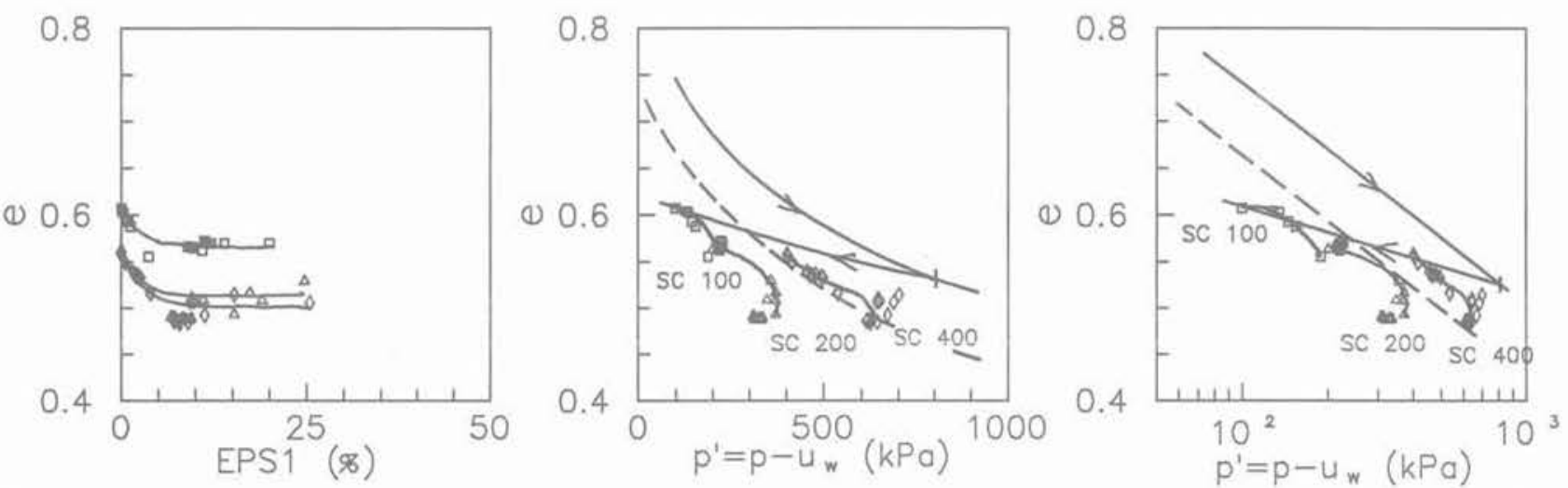

Fig. 6 - Chemin triaxial à pression négative imposée sur le limon de Jossigny surconsolidé par drainage-humidification, à différentes pressions négatives.

Fig. 6 - Triaxial path on Jossigny loam, overconsolidated by drying and wetting. for several controlled negative pressures. 
pente $M=1,3$ : ce point appartient au critère surconsolidé du matériau. Le point extrême de l'essai SC 200 $(\mathrm{OCR}=4)$ semble correspondre aproximativement à l'intersection des critères surconsolidé et normalement consolidé. La valeur de p' pour ce point est voisine de $400 \mathrm{kPa}$, ce qui est en accord avec la contrainte maximale de consolidation de $800 \mathrm{kPa}$. Enfin, pour le dernier essai, à faible degré de surconsolidation $(\mathrm{OCR}=$ 2), le point extrême se place naturellement sur la droite de pente $\mathrm{M}=1,3$. En conclusion, la figure 7 rassemble les résultats de tous les essais à pression positive et négative dans le plan [p'; q].

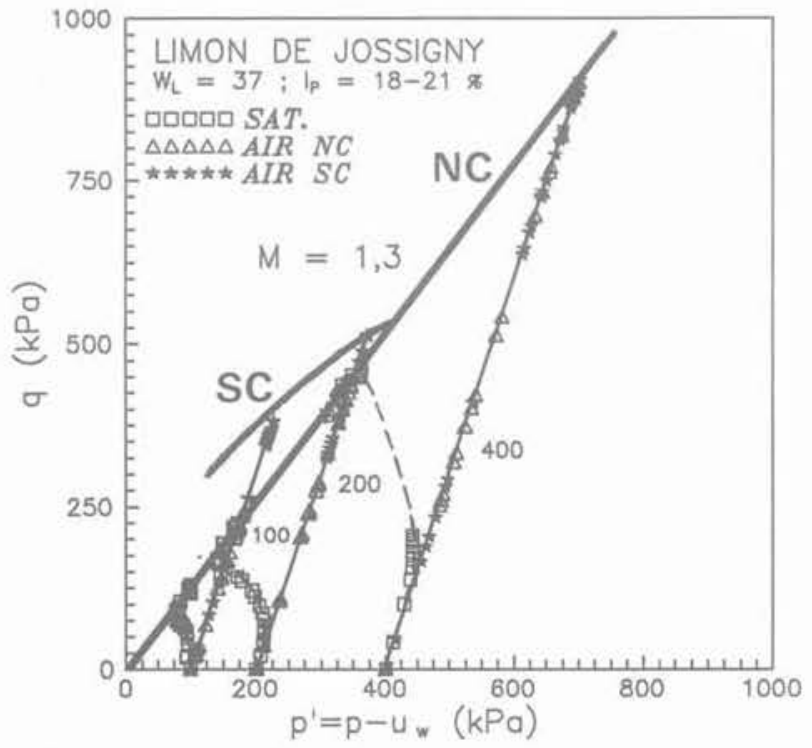

Fig. 7 - Comparaison des chemins à pression positive et négative dans le plan contrainte moyenne effective-déviateur.

Fig. 7 - Comparison between positive and negative pressure paths in the mean effective stress-deviator plane.

Dans le plan [p'; e], les points initiaux des essais à 100 et $400 \mathrm{kPa}$ sont bien disposés sur la courbe de dé. chargement isotrope correspondant à la contrainte de $800 \mathrm{kPa}$. Pendant l'écrasement, les chemins suivent d'abord la courbe de rechargement élastique, définissant ainsi la pseudo-limite élastique volumétrique (BIAREZ et HICHER, 1989), avant de s'en écarter pour rejoindre la courbe de plasticité parfaite définie précédemment. Là encore, l'essai intermédiaire (SC 200) ne suit pas tout à fait cette logique, bien que les points initial et final soient correctement placés. La raison en est pro. bablement un décalage initial du dispositif de mesure placé dans la cellule, la valeur initiale ne coincidant pas avec la mesure faite avant la mise en place.

\section{CONCLUSIONS}

Les différentes séries d'essais sur le limon de Jossigny montrent que la logique des matériaux saturés, de même que la notion de contrainte effective, restent va. lables pour des échantillons à fort degré de saturation soumis à une pression interstitielle d'eau négative ou, ce qui revient au même, à une surpression d'air -.
On retrouve en effet les principales caractéristiques de ces essais et le même critère de plasticité parfaite, tant du point de vue des variations de volume que de la résistance. Ces résultats confirment ceux déjà obtenus sur des matériaux compactés à droite de l'optimum (BIAREZ et al. 1991).

En revanche, lorsque le sol se désature nettement, il n'y a plus équivalence entre la pression négative et une contrainte isotrope puisque les contraintes exercées sur les grains par les ménisques d'eau isolés se traduisent par des forces normales aux plans de contact, qui ne peuvent entraîner de glissement des particules les unes par rapport aux autres, c'est-à-dire de densification du sol. En outre, ces forces de contact confèrent au sol un comportement fragile qui se traduit par des ruptures prématurées sur chemin triaxial, ne permettant pas au matériau d'atteindre la plasticité parfaite.

Le domaine d'application de la notion de contrainte effective, au sens de Terzaghi, apparaît donc limité au domaine des teneurs en eau supérieures approximativement à la limite de retrait; il semble cependant qu'une notion restreinte de contrainte effective, qui exclue le domaine des variations de volume, puisse apporter des simplifications à certains problèmes usuels de mécanique des sols non saturés (BIAREZ et al., 1993).

\section{BIBLIOGRAPHIE}

BIAREZ J., FLEUREAU J.M., ZERHOUNI M.I et SOEPANDJI B.S. (1988), Variations de volume des sols argileux lors de cycles de drainage-humidification. Revue Française de Géotechnique, 41, pp. 63-71.

BIAREZ J., FLEUREAU J.-M. et ZERHOUNI M.I (1989), Compressibilité des sols argileux de $10^{2}$ à $10^{s} \mathrm{~Pa}$. Proceedings of the 12th International Conference on Soil Mechanics and Foundation Engineering, Rio de Janeiro, août.

BIAREZ J., FLEUREAU J.M. et KHEIRBEK-SAOUD S (1991), Validité de $\sigma^{\prime}=\sigma-u_{w}$ dans un sol compacté. Proceedings of the 10th European Conference on Soil Mechanics and Foundation Engineering, Florence, mai, vol. 1, pp. 15-18.

BIAREZ J. et HICHER P.Y. (1989), An introduction to the study of the relation between the mechanics of discontinuous granular media and the rheological behaviours of continuous equivalent media Application to compaction. Proceedings of the 1st International Conference on micromechanics of granular media, Clermont-Ferrand, BIAREZ et GOURVES, eds., Balkema, Rotterdam, pp. 1-13.

BIAREZ J., FLEUREAU J.-M. et TAIBI S. (1993), Modèle microstructural de comportement des sols non saturés. Proceedings of the 13th International Conference on Soil Mechanics and Foundation Engineering, New Delhi, janvier.

DELAGE P., SURAJ DE SILVA G.P.R. et DE LAURE E. (1987), Un nouvel appareil triaxial pour les sols non satures. Proceedings of the 9th European Conference on Soil Mechanics and Foundation Engineering, Dublin, vol. 1, pp. 25-28. 
ESCARIO V. et SAEZ J. (1986), The shear strength of party saturated soils. Géotechnique, $36, \mathrm{n}^{\circ} 13$, pp. 453.456.

FLEUREAU J.M., TAIBI S., SOEMITRO R. et INDARTO (1990), Prise en compte de la pression interstitielle négative dans l'estimation du gonflement. Colloque International «Fondations spéciales , Tlemcen, mars.
FREDLUND D.G., RAHARDJO H. et GAN J.K.M. (1987), Non linearity of strength envelope for unsaturated soils. Proceedings of the 6th International Conference on Expansive Soils, New-Delhi, pp. 49.54.

INDARTO (1991), Comportement mécanique et compactage des matériaux de barrage. Thèse de Doctorat de l'Ecole Centrale de Paris, 4 septembre 1991. 